\title{
Influence des Pressions Anthropiques sur la Dynamique Paysagère de la Reserve Partielle de Faune de Dosso (Niger)
}

\author{
Idrissa Kindo ABDOU ${ }^{1 *}$, Tougiani ABASSE ${ }^{2}$, Moussa MASSAOUDOU², \\ Habou RABIOU $^{3}$, Idrissa SOUMANA ${ }^{2}$ et Jan BOGAERT ${ }^{4}$ \\ ${ }^{I}$ Faculté des Sciences et Techniques, Université Abdou Moumouni de Niamey, \\ ${ }^{2}$ Département de Gestion des Ressources Naturelles, Institut National de la Recherche Agronomique du Niger, \\ Niamey \\ ${ }^{3}$ Université de Diffa \\ ${ }^{4}$ Université de Liège, Gembloux Agro-Bio Tech, Unité Biodiversité et Paysage, Gembloux, Belgique. \\ *Auteur correspondant ; E-mail: aidrissakindo35@yahoo.com; Tel : +2279944 4216
}

\section{RESUME}

La Réserve Partielle de Faune de Dosso est une aire protégée à statut de zone tampon du parc national du W du Niger. De sa création (1962) à aujourd'hui, la réserve est devenue l'espace d'accueil des réfugiés écologiques (migrants agricoles et éleveurs peulhs) du Niger et des pays limitrophes. Une destruction massive de son potentiel ligneux et faunique a été observée ces trois décennies, décriées par les gestionnaires forestiers. Cette étude vise à analyser la dynamique du paysage de la réserve couvrant la période 1986 à 2016. Les images Landsat 5 TM (1986) et Landsat 8 OLI (2016) ont servi de source de données pour cette étude. La classification supervisée par maximum de vraisemblance et le calcul d'indices écologiques ont permis de quantifier la dynamique d'occupation du sol et de détecter les transformations spatiales survenues en 30 ans, au moyen des logiciels ENVI 7.1 et Arcgis 10.2. Les résultats montrent globalement une forte progression des classes d'occupation du sol champs-jachères et sols nus contre une nette régression des classes de formations forestières naturelles. Les transformations spatiales en cours sont la fragmentation, la dissection et la perforation des classes de végétation naturelle. Cette étude a permis de mettre en lumière les processus de transformation spatiale en cours dans le paysage de la réserve et de déterminer les indicateurs d'état pouvant servir de base à la gestion et à la conservation des ressources forestières de la réserve de partielle de faune de Dosso au Niger.

(C) 2019 International Formulae Group. All rights reserved

Mots clés: Aire protégée, composition, configuration, fragmentation, structure spatiale, transformation paysagère.

\section{Influence of Anthropic Pressures on the Landscaping Dynamics of the Partial Reserve of Dosso Fauna (Niger)}

\begin{abstract}
The Dosso Wildlife Reserve is a protected area with buffer zone status in Niger's W National Park. From its creation (1962) to today, the reserve has become the reception area for environmental refugees (agricultural migrants and Fulani pastoralists) from Niger and neighboring countries. A massive destruction of its ligneous
\end{abstract}


and faunal potential has been observed these three decades, decried by the forest managers. This study aims to analyze the landscape dynamics of the reserve covering the period 1986 to 2016. The Landsat 5 TM (1986) and Landsat 8 OLI (2016) images served as a data source for this study. Maximum likelihood-based classification and ecological index computation were used to quantify land cover dynamics and to detect spatial transformations over 30 years using ENVI 7.1 and Arcgis 10.2 software. Overall, the results show a sharp increase in land-fallow and bare soil occupation classes against a clear regression of the natural forest formation classes. The spatial transformations in progress are fragmentation, dissection and perforation of the vegetation classes natural. This study shed light on the spatial transformation processes underway in the reserve landscape and to determine the state indicators that can be used as a basis for the management and conservation of the forest resources of the partial wildlife reserve from Dosso to Niger.

(C) 2019 International Formulae Group. All rights reserved

Keywords: Protected area, composition, configuration, fragmentation, spatial structure, landscape transformation.

\section{INTRODUCTION}

La question de l'exploitation des ressources naturelles en périphérie des aires protégées en Afrique est au cœur des préoccupations des multiples acteurs (Etats, Organismes internationaux, élites intellectuelles et scientifiques, chefferies, agropasteurs, éleveurs) intervenant dans ce genre de milieux (Gueguim et al., 2018; Sadda et al., 2016 ; Mahamadou et Boureima, 2014). $\mathrm{Au}$ Niger, l'étendue des aires protégées couvre environs 8,4 millions d'hectares, soit $6,6 \%$ de la superficie du territoire national (UICN, 2010). Ces aires protégées sont globalement représentatives de l'ensemble des écosystèmes du pays. Ces espaces de conservation et leur périphérie proche correspondent généralement aux zones les plus riches en termes de biodiversité. Par ailleurs, la plupart des aires protégées nigériennes sont fragiles et vulnérables (UICN, 2010) et sont menacées par la sécheresse, la désertification et la pression anthropique entraînant une érosion des ressources génétiques, une perte de capacités de production et de régénération avec comme conséquence, la disparition de certaines espèces (UICN, 2010). A ce jour, plus de $60 \%$ du territoire national ont presque atteint le seuil critique de dégradation des habitats et plus de vingt espèces ont disparu ou sont au bord de l'extinction au Niger (Oryx, Addax, Autruche, etc.) (MEE/LCD, 2010 ; Zean et al., 2018). Les statistiques sur l'état de la conservation $\mathrm{du}$ patrimoine faunique font défaut. Le potentiel actuel est estimé à environ $10 \%$ de ce qui existait, il y a 40 ans (UICN, 2010). Les connaissances restent limitées pour la plupart des espèces animales (mammifères, oiseaux, reptiles, amphibiens, poissons, invertébrés, etc.) ainsi que pour les habitats naturels. Pour des raisons évidentes de conservation des richesses faunistiques et floristiques, des efforts timides d'identification et de protection de certaines aires particulières ont été menées (UICN, 2010).

Une dynamique paysagère s'observe et s'installe dans le temps et dans l'espace de par l'occupation du sol et la diversité biologique qu'abritent ces espaces protégés. Cette dynamique paysagère pourrait ainsi être mise en évidence et quantifier par l'analyse de la composition et la configuration de ses éléments (Bamba, 2010).

Le paysage de la réserve partielle de faune de Dosso (RPFD) n'est pas en marge de toute transformation spatiale au regard de la multiplicité des pressions et menaces dont il fait l'objet. Selon Dan Guimbo (2012), plusieurs espèces sont déclarées menacées de disparition dans la zone périphérique du parc national du W (aire centrale de la réserve de biosphère transfrontalière $\mathrm{du} \mathrm{W}$ du Bénin, du Burkina Faso et du Niger) dont la RPFD lui est contiguë. Cette réserve est classée à la catégorie VI de l'Union Internationale de la Conservation de la Nature (UICN), ne bénéficiant actuellement, d'aucun financement extérieur, rendant son niveau de gestion faible. Pourtant l'aire protégée(RPFD) 
continue à jouer son rôle de zone tampon pour le parc national du $\mathrm{W}$ du Niger. En dépit du classement de cette zone il n'existe pas encore de dispositions, ni d'organe de gestion de cette réserve. L'exploitation agricole et le surpâturage sont généralisés et occasionnent une forte fragmentation de l'aire avec comme conséquence une réduction très sensible de sa superficie, la perte d'habitat et la disparition de certaines espèces. On sait cependant que son état de dégradation est très avancé (UICN, 2010). Mais les informations scientifiques sur l'état de dégradation et le niveau de fragmentation de cette aire bien qu'importante ne sont pas encore disponibles. L'objectif de la présente étude était d'analyser la dynamique du paysage de la RPFD intervenus au cours des 30 dernières années (1986 à 2016).et de déterminer les indicateurs d'état pouvant servir de base à la gestion et à la conservation des ressources forestières de la réserve de partielle de faune de Dosso au Niger.

\section{MATERIEL ET METHODES}

\section{Présentation de la zone d'étude}

La réserve partielle de faune de Dosso est située entre $11^{\circ}$ et $12^{\circ}$ de latitude Nord et $2^{\circ}$ et $3^{\circ}$ de longitude Est, sur deux régions administratives du Niger: Dosso et Tillabéry (Carte 1). Elle a été créée pour servir de zone tampon pour le parc national du $\mathrm{w}$ du Niger avec un statut de conservation de la faune sauvage et de son habitat. Elle couvre une superficie de 306500 ha dont la majeure partie se trouve dans la région de Dosso. Le climat de la région est de type tropical sec et appartient au centre d'endémisme sahélosoudanien avec pluviométrie moyenne annuelle de $800 \mathrm{~mm}$ dans le département de Gaya (extrême Sud de Dosso) et moins de 400 $\mathrm{mm}$ au nord dans le département de Dogondoutchi (extrême Nord de Dosso) (SRAT, 2015). La végétation est composée essentiellement de brousses tigrées, de savanes (arbustives et arborées) et de steppes arbustives. Ces formations couvrent une superficie totale de 2276500 ha soit $16 \%$ des formations naturelles du pays (SRAT, 2015).

\section{Méthodes}

\section{Acquisition des données}

Les images satellites utilisées pour cette étude sont de type Landsat 5 TM (datant du 31 décembre 1986) et Landsat 8 OLI (datant du 31 décembre 2016). Ces images ont été téléchargées gratuitement via le site gouvernemental de United States of Geological Survey (https://www.usgs.gov/software/earth-

explorer-ee.) dans l'archive Landsat grâce aux coordonnées suivantes de la zone d'étude Path 192 et Row 051 et 052.

\section{Prétraitement et classification des images}

Les opérations de prétraitement ont consisté aux mosaiques deux à deux des bandes spectrales grace au module «Mosaicking » du logiciel ENVI 4.7 contenu dans le menu «Basic tools ». Des opérations de redimensionnement ont été ensuite effectuées afin de ramener les dimensions des images mosaiquées à des dimensions de la taille de notre zone d'étude. Des compositions fausses couleurs ont été par la suite composées avec les bandes spectrales 5,4,2 dans le canal rouge, le canal vert et le canal bleu ( Figure 1). Les valeurs radiométriques de ces zones de réflectance extrêmes sont utilisées pour effectuer un stretch linéaire des différentes bandes spectrales (Barima, 2009).

\section{Définition des classes d'occupation du sol}

Neufs classes d'occupation du sol retenues ont été définies sur la base des missions de terrain, plus les données secondaires collectées auprès des institutions de recherche ainisi que par l'analyse visuelle des images landsat acquises correspondant au deux dates d'étude. Il s'agit des cours d'eau (Fleuve Niger; Mare), des formations forestières naturelles (Brousse Tigrée Dégradée; Brousse Tigrée Régulière ; Galerie Forestière; Savane), des agrosystèmes (Mosaïque Champs -Jachère ; Sol -Nu) et des terrains rocheux (Affleurement Rocheux).

\section{La classification des images}

Deux méthodes de classification des unités d'occupation du sol ont été retenues pour la zone d'étude. Il s'agit premièrement 
de la méthode basée sur la classification supervisée avec l'algorithme de «maximum de vraissemblance» (Sadda et al.,2016; Illiassou et al., 2015 ;Mas, 2000) et secondairement l'approche basée sur la digitalisation des unités paysagères de la réserve sur ArcMap (Arcgis 10.2) (Gueguim et al., 2018; Bamba et al., 2008; Casado, 2007).

\section{Validation de la classification supervisée des images de 1986 et 2016}

L'image satellite de 2016 a servi d'image de référence pour valider la classification des unités d'occupation du sol de l'image 1986. Un maximum de zones d'entrainement a été échantillonné dans chaque type ou classe d'occupation du sol afin de rehausser la précision globale «overall accurency » de la classification de l'image de référence.

Pour décrire les changements survenus dans le contexte de notre zone d'étude, il a été établi une matrice de transition entre les deux périodes d'étude $\mathrm{t}_{0(1986)}$ et $\mathrm{t}_{1(2016)}$ (Diallo et al., 2011; Barima et al., 2009).

\section{Calcul des indices de composition et de configuration du paysage}

La structure du paysage est caractérisée pour chaque classe $\mathrm{j}$ d'occupation du sol à partir du nombre de tache $\left(\mathrm{n}_{\mathrm{ij}}\right)$, de l'aire $\left(\mathrm{a}_{\mathrm{ij}}\right)$ et $\mathrm{du}$ périmètre $\left(\mathrm{p}_{\mathrm{ij}}\right)$ des taches de la classe (Diallo et al., 2011). Les indices suivants ont été calculés pour chaque classe d'occupation du sol :

Indice de forme

La forme des taches a été déterminée par le calcul de l'indice de forme à partir de la formule utilisée par Bogaert (2000).

$$
I F_{i}=\frac{a_{i}}{p_{i}{ }^{2}} \quad \text { Equation (1) }
$$

Avec $a_{i}$ l'aire de la tache $i$ et $p_{i}$ le périmètre de la tache $i$ et $I F_{i}$ est l'indice de forme de la tache $i$. Il est sans unité (Bamba, 2010).

Le nombre total de taches d'une classe est la somme des taches qui composent cette classe (Barima, 2009).
L'aire totale $a_{t j}$ d'une classe $j$ est la surface totale occupée par l'ensemble des taches $i$ qui composent cette classe. Exprimée dans le cas présent en $\mathrm{Km}^{2}$. Sa formule est la suivante :

$$
a_{t j}=\sum_{i=1}^{n} a_{i j} \quad \text { Equation (2) }
$$

Où $a_{i j}$ est la i-ème aire de la classe $\mathrm{j}$

La proportion des aires des classées a été calculée par la formule qui suit :

$\mathrm{P}(a j)=\frac{a_{j}}{a_{t}}$ exprimée en $\% \quad$ Equation (3)

La dominance $\mathrm{D}\left(a_{j}\right)$ indiquant la proportion d'aire occupée par la tache dominante dans la classe $j$ a aussi été prise en compte. Il s'agit de la part occupée dans l'aire totale $\left(a_{t j}\right)$ par la plus grande tache de la classe $j$ notée $a_{\operatorname{maxj}}$ (Diallo et al., 2011).

$$
\begin{aligned}
& \mathrm{D}\left(a_{j}\right)=\frac{\boldsymbol{a}_{\max , j}}{\boldsymbol{a}_{t j}} \quad \text { Equation (4) } \\
& 0<\mathrm{D}(a j) \leq 100 . \text { Plus la valeur de la }
\end{aligned}
$$
dominance est grande, moins la classe est fragmentée.

Le périmètre total $P_{t j}$ d'une classe $\mathrm{j}$ est la somme des contours des taches $i$ qui composent cette classe. Exprimé ici en Km. Il se calcule par la formule suivante :

$$
P_{t j}=\sum_{i=1}^{n} P_{i j} \quad \text { Equation (5) }
$$

La dimension fractale permet de quantifier le degré d'irrégularité et de fragmentation d'un objet (Bamba, 2010). En effet, les objets naturels sont caractérisés par des formes complexes avec une valeur de dimension fractale élevée $(D f \approx 2)$ tandis que les objets modelés par l'homme ont des formes caractéristiques régulières (carré, rectangle...) et une valeur de dimension fractale faible $(D f \approx 1)$ (Bamba, 2010). Elle varie de 1 à 2 (Diallo, 2011).Elle est déterminée par la relation qui existe entre le périmètre et l'aire de l'ensemble des taches de la classe :

$$
P_{i j}=k a_{i j} \frac{D_{f j}}{2} \quad \text { Equation (6) }
$$

Avec $P_{i j}$ la somme totale du contour de chacune des taches d'une classe.

Soit, en utilisant une transformation logarithmique (Diallo, 2011):

$\log \mathrm{p}_{\mathrm{ij}}=\log \mathrm{k}+(\mathrm{Dfj} / 2) \cdot \log \mathrm{a}_{\mathrm{ij}} \quad$ Equation (7) $\mathrm{Df}_{\mathrm{j}}$ est la dimension fractale de la classe $\mathrm{j}$ et $\mathrm{k}$ une constante. 


\section{Mise en évidence des changements}

Le processus de transformation spatial a été défini à l'aide d'un arbre de décision proposé par (Bogaert et Hong, 2004) (Figure 2).

Les transformations du paysage retenues sont : l'agrégation (fusion de taches), la suppression (disparition de taches), la création (formation de nouvelles taches), la déformation (changement de forme des taches), l'agrandissement (expansion de taille des taches), la perforation (formation de trous dans les taches), le déplacement (translocation des taches) le rétrécissement (réduction de taille des taches), la fragmentation (rupture de la continuité en plusieurs taches disjointes) et la dissection (subdivision des taches par des lignes de petite dimension).

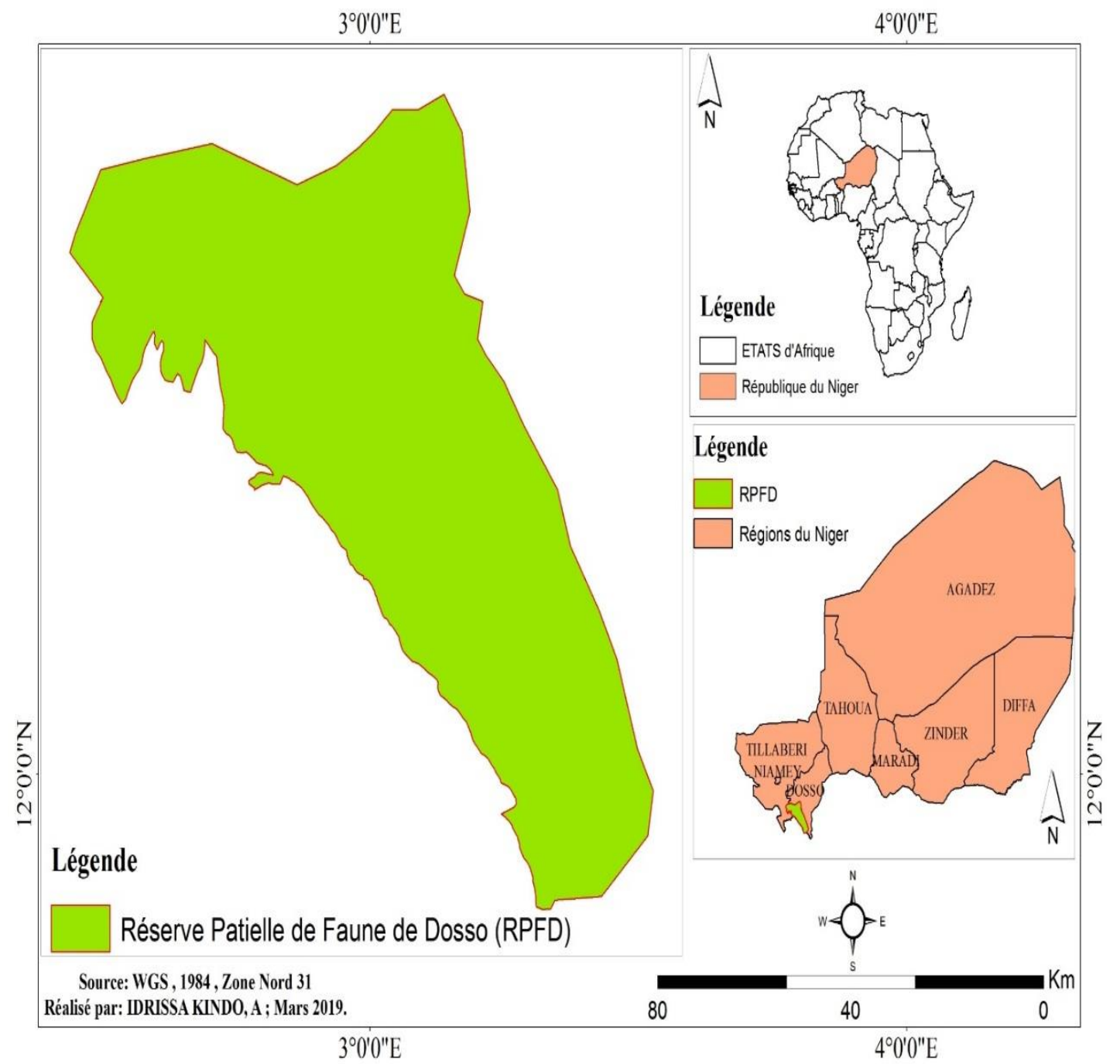

Carte 1 : Localisation administrative de la réserve partielle de faune de Dosso. 


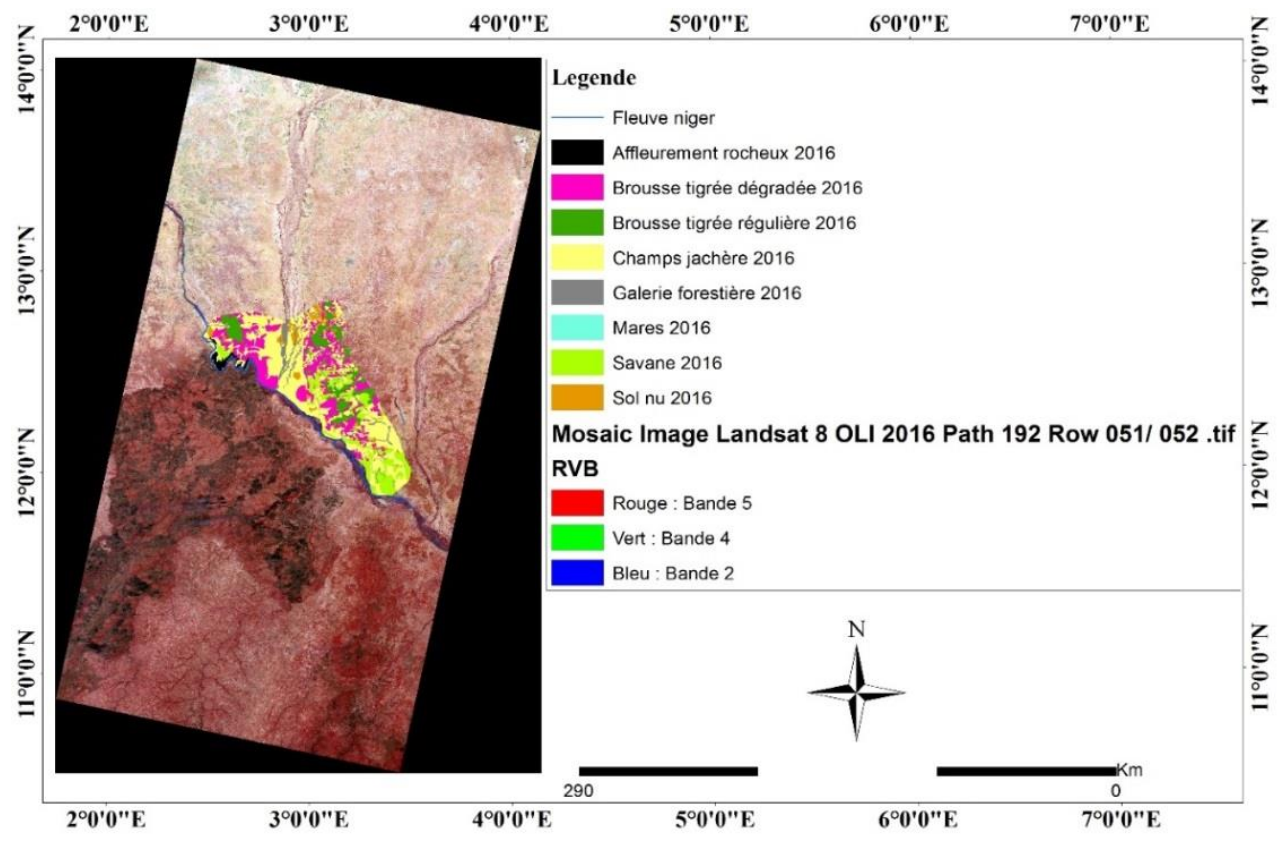

Figure 1: Mosaïque d'images Landsat 8 OLI de 2016 de la zone d'étude.

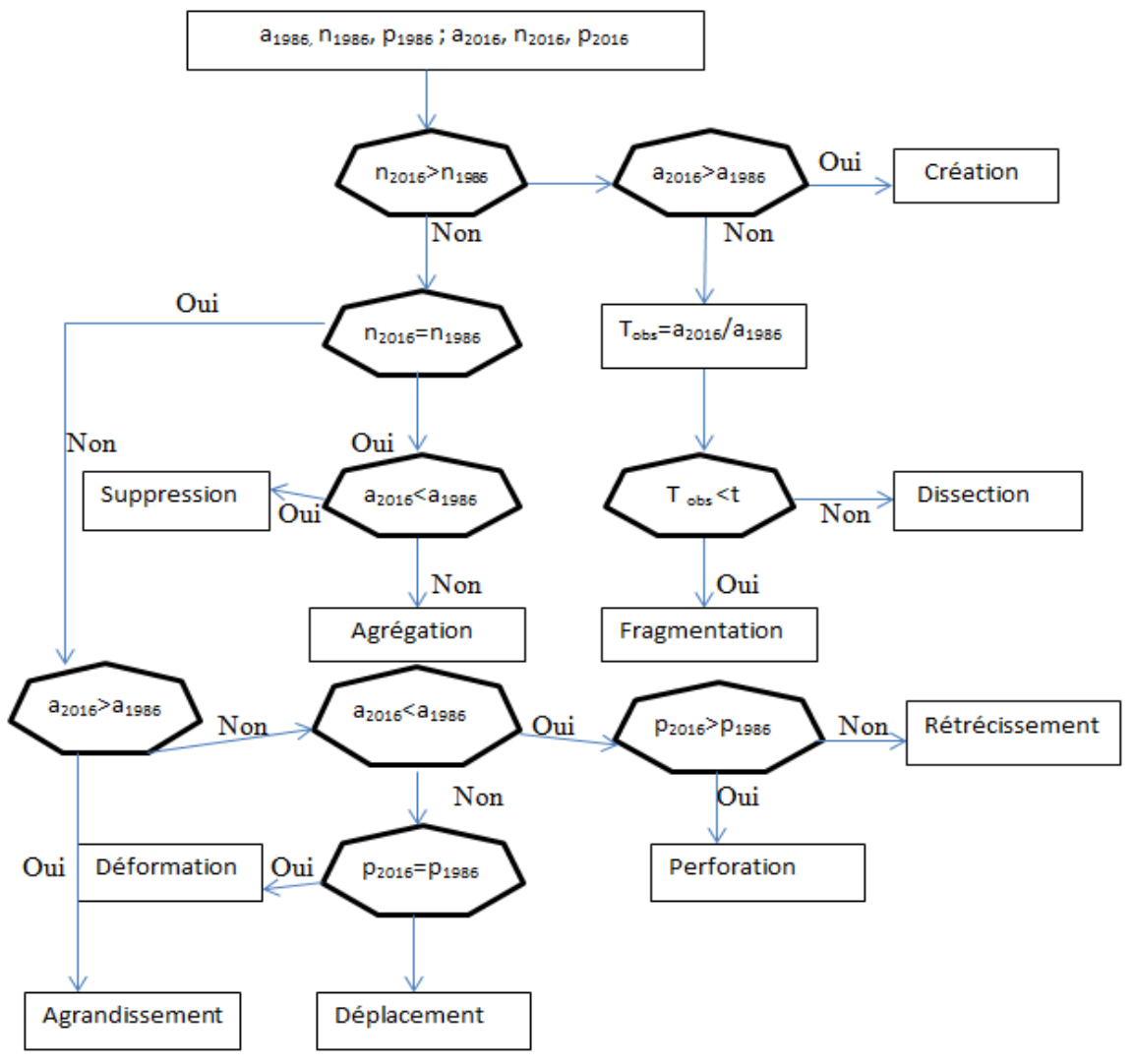

Figure 2 : Identification du processus de transformation spatiale. 


\section{RESULTATS \\ Vérification de la classification des images de 2016}

Le Tableau 1 présente la matrice de confusion ou tableau de contingence des unités d'occupation du sol de la zone d'étude. Les nombres situés sur la diagonale (Tableau 1) correspondent aux pourcentages de pixels bien classifiés. On constate que l'ensemble des classes d'occupation du sol présentent une précision de $100 \%$. La précision globale obtenue à partir de l'équation 1 est de $100 \%$. La valeur de coefficient kappa obtenue à partir de l'équation 2 est de 1. Cette valeur indique une bonne qualité de la classification comme l'illustrent les deux cartes d'occupation du sol (Figures 3 et 4).

\section{Evaluation de la dynamique de l'occupation du sol entre 1986 et 2016}

Le Tableau 2 présente la matrice de transition des changements d'occupation de sol survenus en trois décennies (entre 1986 et 2016) au niveau de la réserve partielle de faune de Dosso. On constate que le taux de brousse tigrée dégradée dans le paysage est passé de $18 \%$ en 1986 à $30 \%$ en 2016. Le taux de la brousse tigrée régulière qui était de $12,13 \%$ en 1986 est tombé à 8,46\% en 2016. On remarque que la savane qui constituait la matrice du paysage de la réserve partielle de faune de Dosso avec un taux de $44,4 \%$ en 1986 a chuté en 2016 avec un taux de 14,9\%. $\mathrm{La}$ nouvelle matrice $\mathrm{du}$ paysage est aujourd'hui la mosaïque champ-jachère avec un taux de $33,8 \%$ en 2016 , contre $18,4 \%$ en 1986. On constate une augmentation du complexe sol nu-habitat qui était de $1,5 \%$ en 1986, contre $5,89 \%$ en 2016. On remarque aussi une légère hausse du taux de la mosaïque galerie forestière-mare passant de 4,56\% en 1986 à 5,80\% en 2016. De façon générale, on constate que les taux d'occupation des classes brousse tigrée dégradée, mosaïque champs jachère et le complexe sol nu -habitat ont augmenté entre 1986 à 2016. Cette augmentation s'est faite principalement au détriment de la brousse tigrée régulière et la savane. Les taux de $33,8 \%$ et $30 \%$ qu'occupent actuellement les mosaïques champs-jachère et la brousse tigrée dégradée, respectivement de $15,4 \%$, à 9,9\% de savane et $4,4 \%$ de brousse tigrée régulière ont été affectées aux mosaïques champs-jachère et à la brousse tigrée dégradée entre 1986 et 2016. Ainsi, la classe savane et la classe brousse tigrée régulière ont constitué ces dernières décennies les deux classes principales pourvoyeuses d'espace agricole dans le paysage de la réserve partielle de faune de Dosso de 1986 à 2016. Les sols nushabitats ont contribué à hauteur de $0,90 \%$ pour la mosaïque champs-jachère et $0,27 \%$ pour la brousse tigrée dégradée. Le complexe galerie forestière -mare a aussi contribué en espace à hauteur respective de $0,33 \%$ pour la brousse tigrée dégradée, $0,03 \%$ pour la brousse tigrée régulière, $0,30 \%$ pour la savane, $0,60 \%$ pour la mosaïque champsjachère et $0,09 \%$ pour la mosaïque sol nuhabitat.

\section{Analyse quantitative de la structure spatiale du paysage: Composition et configuration}

L'analyse des indices de structure spatiale a permis d'inférer les transformations spatiales survenues en 30 ans. Ainsi, on constate une augmentation du nombre de taches pour l'ensemble des classes d'occupation du sol retenues. Le changement identifié est la fragmentation avec un morcellement des taches initiales. Ainsi pour les classes de brousses tigrées dégradées et régulières, de savanes et de mosaïques champs jachères, leur nombre de taches a été multiplié respectivement par $12,8,3$ et 4 . Pour les mosaïques sols nus-habitats, le nombre de taches a été multiplié par 6 tandis que pour le complexe galerie forestière -mare et les affleurements rocheux, leur nombre de taches n'a pas changé en 30 ans. Quant aux aires totales des classes d'occupation du sol, une diminution est remarquée seulement au niveau des classes brousse tigrée régulière et savane entre la période 1986 et 2016 par rapport aux autres classes (Tableau 3). La brousse tigrée régulière a connu une dissection entre 1986 et 2016 car le ratio $t_{\mathrm{obs}}=0,71>\mathrm{t}_{=0,5}$. Pour la savane le ratio $t_{o b s}=0,37<t_{=0,5}$, on parle de 
fragmentation de la savane entre 1986 et 2016. Les classes d'occupation de sols de savanes, de sols nus-habitats et de galeries forestières-mares ont connu une diminution $\mathrm{du}$ périmètre total en 30 ans mais contrairement aux classes de brousses tigrées dégradées, de brousses tigrées régulières, les mosaïques champs jachère, et les affleurements rocheux où une augmentation du périmètre total est observée.

Un processus de perforation est détecté comme changement pour la classe brousse tigrée régulière entre 1986 et 2016 car le périmètre total de 2016 de cette même classe est supérieur à celui de 1986 ainsi que l'aire totale de la même classe en 2016 étant inférieure à celle de 1986.

L'analyse des valeurs de la dominance de la plus grande tache de chaque type d'occupation du sol entre 1986 et 2016 fait remarquer une augmentation de la proportion de la plus grande tache au niveau de la brousse tigrée dégradée de 9,23\% en 1986 à $9,79 \%$ en 2016. La valeur de la dominance concernant la brousse tigrée régulière a été réduite par 2 fois de sa surface passant ainsi de 49,31\% en 1986 à 20,03\% en 2016. Le taux de la plus grande tache de savane est passé de 11,94\% en 1986 à 7,64\% en 2016. Le même constat se dégage au niveau de la classe mosaïque champs-jachère dont la valeur de la dominance de la plus grande tache a chuté de $7,71 \%$ en 1986 à $4,17 \%$ en 2016 . Le complexe sol nu habitat a un taux de l'indice dominance qui est réduit de $48,39 \%$ en 1986 à $10,57 \%$ en 2016 soit près de 4 fois. Les proportions de l'indice de dominance sont respectivement passées de 11,76\% en 1986 à 20,37\% en 2016 (soit une augmentation) pour les classes galerie forestière -mare et de 76,66\% en 1986 à 47,22\% en 2016 (soit une réduction) pour la classe affleurement rocheux.

L'analyse de la dimension fractale de chaque classe d'occupation du sol déterminée entre 1986 et 2016 montre que pour l'ensemble des classes d'occupation du sol les valeurs sont proches de 2 . Cela signifie que les toutes classes présentent des taches de contours complexes durant la période d'étude. Les valeurs de l'indice de forme sont faibles pour toutes les classes entre 1986 et 2016.

Tableau 1: Matrice de confusion obtenue à partir de la classification de l'image landsat 8 Oli de 2016 des différentes unités d'occupation du sol de la RPFD.

\begin{tabular}{llllllllll}
\hline Image classifiée & FN & BTD & BTR & MC-JAC & SN & GF & SAV & AFROC & MAR \\
\hline FN & 100 & 0 & 0 & 0 & 0 & 0 & 0 & 0 & 0 \\
BTD & 0 & 100 & 0 & 0 & 0 & 0 & 0 & 0 & 0 \\
BTR & 0 & 0 & 100 & 0 & 0 & 0 & 0 & 0 & 0 \\
MC-JAC & 0 & 0 & 0 & 100 & 0 & 0 & 0 & 0 & 0 \\
SN & 0 & 0 & 0 & 0 & 100 & 0 & 0 & 0 & 0 \\
GF & 0 & 0 & 0 & 0 & 0 & 100 & 0 & 0 & 0 \\
SAV & 0 & 0 & 0 & 0 & 0 & 0 & 100 & 0 & 0 \\
AFROC & 0 & 0 & 0 & 0 & 0 & 0 & 0 & 100 & 0 \\
MAR & 0 & 0 & 0 & 0 & 0 & 0 & 0 & 0 & 100 \\
\hline Total & 100 & 100 & 100 & 100 & 100 & 100 & 100 & 100 & 100 \\
\hline
\end{tabular}

Légende : FN: Fleuve Niger; BTD : Brousse Tigrée Dégradée; BTR : Brousse Tigrée Régulière; MC-JAC : Mosaïque Champs -Jachère ; SN : Sol-Nu ;GF: Galerie Forestière ; SAV :Savane ; AFROC :Affleurement Rocheux ; MAR : Mare 
Tableau 2 : Matrice de transition (en pourcentage \%) de l'occupation du sol entre 1986 et 2016.

\begin{tabular}{|c|c|c|c|c|c|c|c|c|}
\hline $7_{1986}^{2016}$ & BTD & BTR & SAV & MC-JAC & SN-HAB & SN-HAB & GF-M & AFROC \\
\hline BTD & 13,79 & 0,34 & 0,07 & 1,87 & 1,87 & 0,06 & 0 & 18 \\
\hline BTR & 4,47 & 5,24 & 0,46 & 1,43 & 0,49 & 0,04 & 0 & 12,13 \\
\hline SAV & 9,98 & 2,82 & 12,12 & 15,45 & 2,19 & 1,57 & 0,36 & 44,49 \\
\hline MC-JAC & 1,1 & 0,03 & 1,84 & 13,58 & 0,99 & 0,9 & 0 & 18,44 \\
\hline SN-HAB & 0,27 & 0 & 0,13 & 0,9 & 0,26 & 0,02 & 0 & 1,58 \\
\hline GF-M & 0,33 & 0,33 & 0,3 & 0,6 & 0,09 & 3,21 & 0 & 4,56 \\
\hline AFROC & 0,06 & 0 & 0,04 & 0,01 & 0 & 0 & 0,69 & 0,8 \\
\hline Totaux & 30 & 8,46 & 14,92 & 33,84 & 5,89 & 5,8 & 1,06 & 100 \\
\hline
\end{tabular}

Légende : FN: Fleuve Niger; BTD : Brousse Tigrée Dégradée; BTR : Brousse Tigrée Régulière ; MC-JAC : Mosaïque Champs -Jachère ; SN : Sol-Nu ; GF : Galerie Forestière ; SAV : Savane ; AFROC : Affleurement Rocheux ; MAR : Mare.

Tableau 3 : Indices de structures spatiales calculées pour chaque type d'occupation de sol entre 1986 et 2016.

\begin{tabular}{|c|c|c|c|c|c|c|c|}
\hline 1986 & BTD & BTR & SAV & MC-JAC & SN-HAB & GF-M & AFROC \\
\hline $\mathrm{n}_{\mathrm{j}}$ & 27 & 8 & 116 & 184 & 47 & 59 & 5 \\
\hline $\left.\mathrm{at}_{\mathrm{j}(\mathrm{Km}}{ }^{2}\right)$ & 130 & 752 & 2606 & 1285 & 106 & 216 & 60 \\
\hline $\mathrm{Pt}_{\mathrm{j}(\mathrm{Km})}$ & 335 & 630 & 2939 & 2189 & 197539 & 1163 & 98 \\
\hline $\mathrm{D}_{\mathrm{j}}$ & 9,23 & 49,31 & 11,94 & 7,71 & 48,39 & 11,73 & 76,66 \\
\hline $\mathrm{Df}_{\mathrm{j}}$ & 1,81 & 1,52 & 1,67 & 1,76 & 2,32 & 2,11 & 1,56 \\
\hline $\mathrm{If}_{\mathrm{ij}}$ & 0,00769 & 0,0021 & 3,1511 & 7,848 & $9,36 \mathrm{E}-07$ & 0,00158 & 0,016 \\
\hline If $f_{\text {moy }}$ & 0,000284 & 0,00026 & 0,0271 & 0,0426 & $1,99 \mathrm{E}-07$ & 0,0000267 & 0,0032 \\
\hline 2016 & BTD & BTR & SAV & MC-JAC & SN-HAB & GF-M & AFROC \\
\hline $\mathrm{n}_{\mathrm{j}}$ & 337 & 69 & 309 & 653 & 268 & 79 & 6 \\
\hline $\mathrm{at}_{\mathrm{j}\left(\mathrm{Km}^{2}\right)}$ & 1174 & 534 & 968 & 2086 & 439 & 266,72 & 72 \\
\hline $\mathrm{Pt}_{\mathrm{j}(\mathrm{Km})}$ & 2674 & 840 & 2097 & 4537 & 1206 & 1117 & 118 \\
\hline$D_{j}$ & 9,79 & 20,03 & 7,64 & 4,17 & 10,57 & 20,37 & 47,22 \\
\hline $\mathrm{Df}_{\mathrm{j}}$ & 1,84 & 1,7 & 1,82 & 1,84 & 1,87 & 2,01 & 1,58 \\
\hline $\mathrm{If}_{\mathrm{ij}}$ & 1,64 & 7,56 & 2,2 & 1,01 & 0,25709 & 0,2295 & 0,0051 \\
\hline If $_{\text {moy }}$ & 0,023 & 0,0224 & 0,0071 & 0,00154 & 0,00096 & 0,003 & 0,00085 \\
\hline
\end{tabular}

Légende : FN : Fleuve Niger; BTD : Brousse Tigrée Dégradée ; BTR : Brousse Tigrée Régulière ; MC-JAC : Mosaïque Champs -Jachère ; SN : Sol-Nu ; GF : Galerie Forestière ; SAV : Savane ; AFROC : Affleurement Rocheux ; MAR : Mares. $\mathrm{n}_{\mathrm{i}}$ : nombre totale de taches d'une classe $\mathrm{i}$; $\mathrm{at}_{\mathrm{j}}$ : aire totale d'une classe $\mathrm{j} ; \mathrm{Pt}_{\mathrm{j}}$ : périmètre totale d'une classe $\mathrm{j} ; \mathrm{D}_{\mathrm{j}}:$ Dominance de la plus grande tache dans l'aire totale $(\mathrm{en} \%) ; \mathrm{Df}_{\mathrm{j}}$ : Dimension fractale de la classe $\mathrm{j} ; \mathrm{If}_{\mathrm{ij}}$ : Indice de forme de la tache dans la classe $\mathrm{j}$; If $\mathrm{Ifoy}_{\text {m }}$ : Indice de forme moyen de la classe $\mathrm{j} ; \mathrm{Km}^{2}$ (Kilomètre carré) ; Km (Kilomètre) . 


\section{DISCUSSION}

\section{Influence de la mise en culture sur la dynamique de la composition du paysage}

L'analyse de la matrice de transition a révélé au cours de ces trois décennies une transformation majeure de la composition du paysage de la RPFD avec comme conséquence le changement de matrice du paysage (Figures 3 et 4). Cette modification de l'occupation des sols de la RPFD a été engendrée par l'effet de la mise en culture croissante ces dernières années combinée aux effets de l'exploitation anarchique des produits forestiers ligneux (bois de feu et de service) et non ligneux (écorchage, cueillettes de fruits et feuilles) mais aussi de surpâturage pratiqué par les agropasteurs et les transhumants peulhs. En effet, plusieurs auteurs ont rapporté que les modifications de la composition des paysages dans les zones sahélo-soudanienne, soudanienne, soudanoguinéenne et guinéenne sont l'œuvre d'une anthropisation rapide et progressive manifestée par des pratiques agro-sylvopastorales non durables (Alohou et al., 2016 ; Alongo et al., 2013; Illiassou et al., 2015; Mama et al., 2014). L'effet de la pression anthropique sur la dynamique du paysage de la RPFD s'est traduit en 30 ans par une extension considérable du complexe champjachère (Figure 5) dans la réserve au détriment de la savane et de la brousse tigrée régulière qui connaissent aujourd'hui une régression importante de leurs aires respectives. Cette tendance s'explique par les défrichements observés dans la zone et touche pratiquement l'ensemble des unités d'occupation des sols. Les causes à l'origine de cette transformation spatiale sont liées à l'arrivée massive ces dernières années, des migrants agricoles et éleveurs venant de diverses localités du pays et d'autres nationalités (Bénin et Mali). Les raisons de ces déplacements vers la RPFD sont surtout liées à la saturation des terres agricoles de leurs localités respectives et le manque de pâturages. Les événements climatiques extrêmes et récurrents caractérisés par des années sèches et la croissance démographique ont occasionné des déplacements massifs des populations vers la réserve (Sadda et al., 2016; Kabirou, 2013; Bachir, 2013; Sitayeb et Benabdeli, 2008). Cette migration massive de populations autochtones et allochtones vers l'aire protégée nécessite de défricher davantage de champs dans les espaces considérés comme réserves forestières à vocation sylvo-pastorale (Kabirou, 2012) Cette réserve regorgeait dans le temps de ressources naturelles disponibles et dont les populations locales jouissaient de l'usufruit. Ainsi, la satisfaction des besoins en terre de cultures, de pâturages et des produits forestiers ligneux et non ligneux par les populations autochtones et allochtones ont considérablement augmenté ces dernières années, avec pour conséquence immédiate , une modification de la composition et la structure spatiale de la réserve. En 30 ans, la matrice du paysage (Figure 6) est passée de la brousse tigrée régulière, de savane au complexe champ jachère suivi de la création de tache de brousse tigrée dégradée, de sols nus-habitation. Les mares ont diminué en nombre et en surface. Cette situation s'explique par la recomposition généralisée de l'occupation des sols de la réserve ces dernières années avec une modification des chenaux d'écoulements, sources d'appauvrissement des mares. Les taux de régression constatés respectivement au niveau de la brousse tigrée régulière et les savanes sont de $(-8,9 \%)$ et $(-17,01 \%)$ en 30 ans soit un taux annuel respectif de l'ordre de ($0,29 \%)$ et $(-0,56 \%)$. Selon Tente (2005), lorsqu'une brousse tigrée régulière passe à une brousse tigrée dégradée, très dégradée ou à un champ de culture, on parle respectivement de régression d'ordres 1, 2 ou 3. On estime d'après FAO (2001), une diminution du couvert forestier au Niger de l'ordre de 3,7\% par an. Selon Larwanou (2005), cette perte de couvert forestier est imputable à l'action de l'homme sur son milieu au sahel. Cette situation trouve son explication dans l'accroissement rapide de la population (3,9\% selon INS, 2012 ; Bamba et al., 2010; Moussa, 2016; Atachada et al., 2018). 


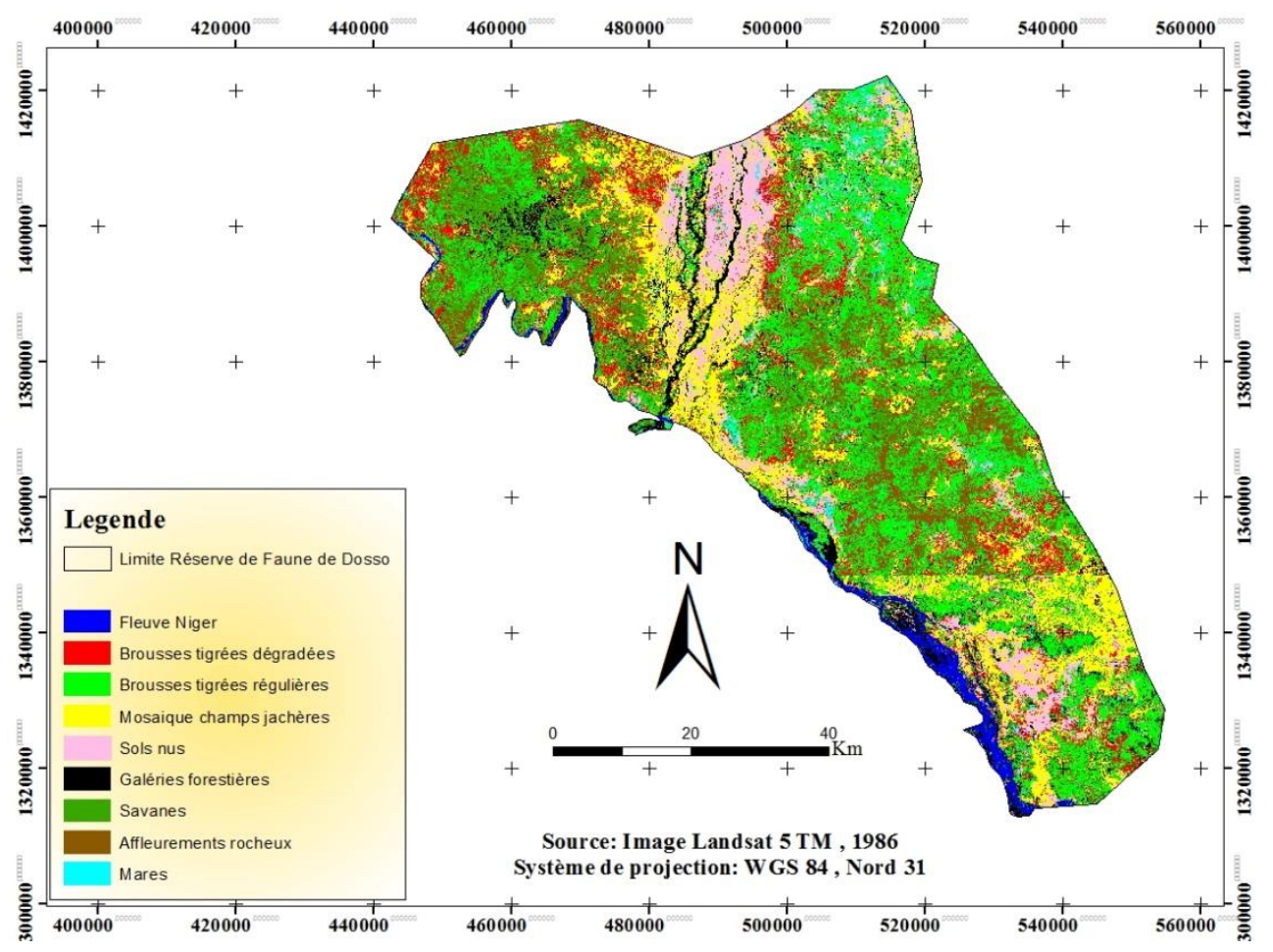

Figure 3: Carte d'occupation des sols obtenue à partir de la méthode de classification supervisée sous Envi 4.7 (images Landsat5 TM de 1986).

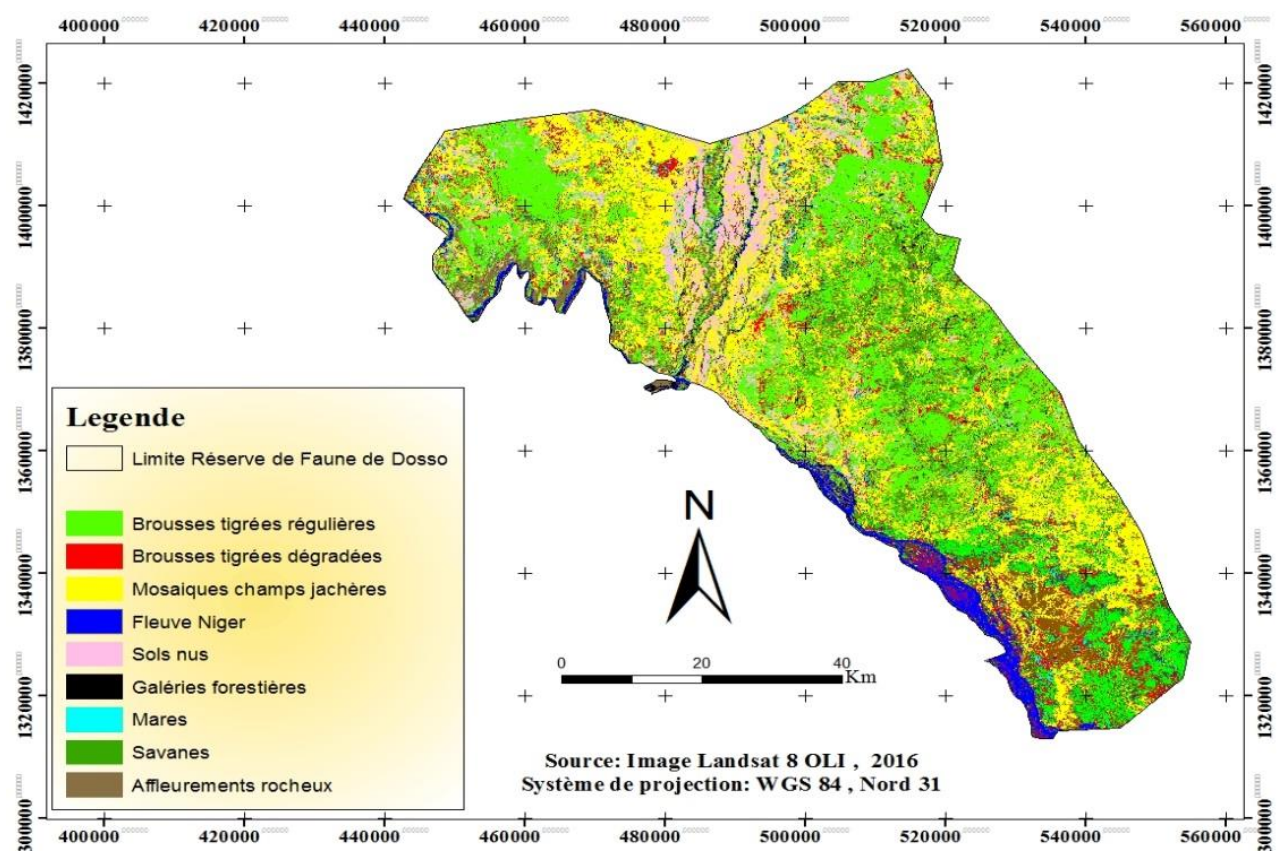

Figure 4: Carte d'occupation des sols obtenue à partir de la méthode de classification supervisée sous Envi 4.7 (images Landsat 8 OLI 2016). 


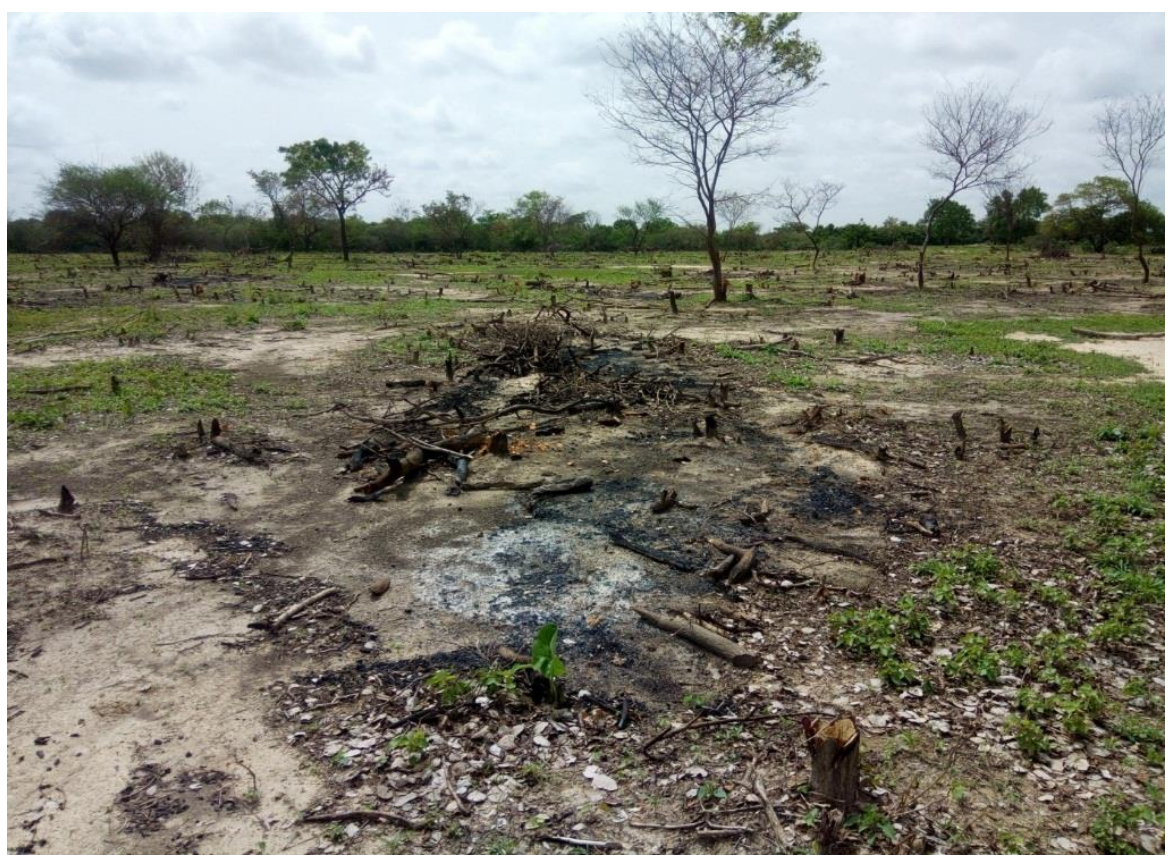

Figure 5: Nouveau défrichement sur brulis de la brousse tigrée régulière en champs pour la mise en culture en début de saison de pluie (cliché Abdou, 2017).

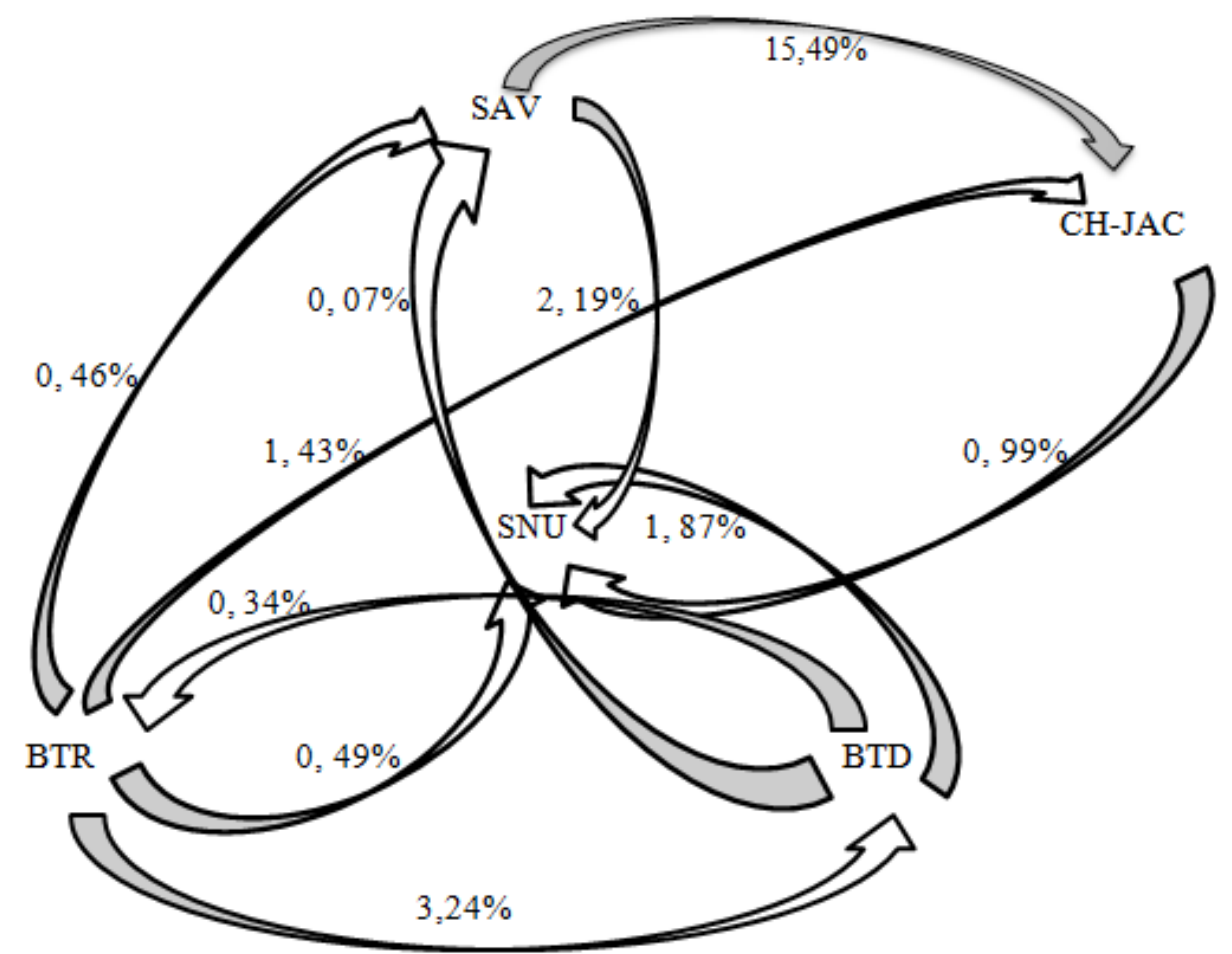

Figure 6. Diagramme de transfert (pourcentage \%) d'occupation du sol dans le paysage de la réserve entre 1986 et 2016.

Légende: Brousses Tigrées Régulières(BTR); Brousses Tigrées Dégradées (BTD); Champs Jachères (CH-JAC); Savanes (SAV); Mares (MAR); Sols Nus (SNU); Galerie Forestière (GF); Habitations (HAB). 


\section{Conclusion}

L'étude a permis de mettre en lumière la dynamique des unités d'occupation des sols de la RPFD entre 1986 et 2016 au moyen de la télédétection spatiale et le système d'information géographique (SIG). En 30 ans la RPFD a subi une modification substantielle de son occupation des sols due en partie aux actions anthropiques, manifestée dans ce travail, par le calcul d'un certains nombres d'indices de structure spatiale retenus, employés en écologie du paysage. L'étude a révélé aussi une régression nette, de la brousse tigrée régulière et de la savane, qui constituaient auparavant la matrice dominante de la RPFD, au profit de la brousse tigrée dégradée, du complexe champ jachère et des sols nus-habitation. En ce qui concerne les galeries forestières elles ont connu une légère amélioration. Quant aux mares, elles ont diminué en surface et en nombre. Pour ce qui est des affleurements rocheux, ils sont pratiquement stables. En effet, l'étude de la structure spatiale (par le biais des indices écologiques) a montré les processus de transformation spatiale en cours dans la RPFD. Il s'agit de la création de nouvelles taches de brousse tigrée dégradée, des champs jachères, de sols nus et des habitations; la dissection et la perforation de la brousse tigrée régulière; la fragmentation de la savane et une suppression des mares. Au regard de ces résultats liminaires, obtenus sur la dynamique spatio temporelle de l'occupation des sols, il s'avère important de conduire une étude de caractérisation de la phyto diversité des différentes classes d'occupation des sols afin d'évaluer la dynamique mais aussi d'apprécier les menace anthropiques, le tout dans une perspective d'aménagement et de gestion durable des ressources végétales de la RPFD.

\section{CONFLIT D'INTERETS}

Les auteurs de cette étude déclarent qu'aucun conflit d'intérêts n'a été signalé.

\section{CONTRIBUTIONS DES AUTEURS}

IKA a assuré $70 \%$ de ce travail dans les travaux suivants : Conception du protocole de recherche, missions de terrain, conception des cartes, analyse et interprétation des données et rédaction du manuscrit. Les $30 \%$ du travail sont partagés aux auteurs ci-après : TA a contribué au financement de ce travail, à recadrer le travail de terrain et à la lecture initiale scientifique du manuscrit. MM, HR et IS ont soutenus respectivement à la relecture scientifique et critique pour améliorer la qualité de la version finale du manuscrit et JB a validé le protocole de recherche.

\section{REMERCIEMENTS}

Les auteurs remercient le centre national de surveillance écologique et environnemental et le centre régional AGRHYMET pour leurs appuis techniques et la mise à disposition des images satellites. Les auteurs remercient également le Conseil Régional de Dosso ainsi que les directions techniques (régionales et départementales) de l'environnement, de l'agriculture et de l'élevage de la région de Dosso pour l'appui tant administratifs que techniques de terrains.

Les auteurs adressent aussi également leurs remerciements aux personnes ressources suivantes: Professeur ABASSE Tougiani pour sa contribution financière à la réalisation de ce travail ainsi qu'au Dr Salamatou Abdouramane, pour son appui à la réalisation des cartes.

\section{REFERENCES}

Atchada CC, Zoffoun AG, Akplo AHA, Tente AB, Djego JG. 2018. Mode d'utilisation des terres et stock de carbone organique du sol dans le bassin supérieur de Magou au Bénin. Int. J. Biol. Chem. Sci., 12(6): 2818-2829. DOI: https:// dx.doi.org /10.4314/ijbcs.v12i6.27

Alongo S, Visser M, Drout T, Kombele F, Colinet G, Bogaert J. 2013. Effets de la fragmentation des forets par l'agriculture itinérante sur la dégradation de quelques propriétés physiques d'un Ferralsol échantillonné à Yagambi, R.D. Congo. Tropicultura, 31(1): 36-43.URL http://hdl.handle.net/2268/160233

Alohou EC, Ouinsavi C, Sokpon N. 2016. Facteurs déterminants de la fragmentation du bloc foret classée- 
forets sacrées au Sud - Bénin. Journal of Applied Biosciences, 101:9618-9633. DOI: http://dx.doi.org/10.4314/jab.v101i1.5

Bachir A. 2013. Dynamique de l'occupation $\mathrm{du}$ sol sur le plateau de Dyabou (Département de Say, Niger). Revue Scientifique Semestrielle Territoires, Sociétés et Environnement, 2: 27-36. URLhttp://uam.refer.ne/index.php/recher che/publications-scientifiques/197publication-mars-2014

Bamba I, Mama A, Neuba DFR, Koffo KJ, Traoré D, Visser M, Sinsin B, Lejoly J, Bogaert J. 2008. Influence des pressions anthropiques sur la dynamique spatiotemporelle de l'occupation du sol dans la province du Bas Congo (République Démocratique du Congo). Sciences et Nature, 5 : 4960 . DOI:10.4314/scinat.v 5il.42151

Bamba I. 2010. Anthropisation et dynamique spatio-temporelle de paysages forestiers en RDC. Thèse de Doctorat, Université Libre de Bruxelles, Bruxelles, p205. URL https:// www.congoforum.be/upldocs/Forets $\% 20$ These_finale_IBAMBA.pdf

Barima Y S S, Barbier N, Bamba I, Traoré D, Lejoly J, Bogaert J. 2009. Dynamique paysagère en milieu de transition forêts -savane ivoirienne. Bois et Forêts des tropiques, 299 (1) : 15-25. DOI: 10.19182/bft2009.299.a20419

Bogaert J. 2000. Quantifying habitat fragmentation as a spatial process in a patch-corridor- matrix landscape model. $\mathrm{PhD}$ dissertation, University of Antwerp, Antwerp.

Bogaert J, Hong SK. 2004. Landscape ecology monitoring landscape dynamics using spatial pattern metrics. In Ecological Issues in a Changing World: Status, Response and Strategy,

Hong SK (ed). Kluwer Academic Publishers: Netherlands ; 109-131.

Casado A. 2007. Etude de la structure et de la dynamique des paysages de montagne: Exemple
Du bassin versant de la Voireuze, affluent de l'Alagnon entre 1948 et 2000. Mémoire de Master 2, Université Blaise Pascal, Clermond Ferrand, p 51. URL geolab.univ-

bpclermont.fr/IMG/pdf/These_Ana1.pdf (page consultée le 28/02/2018).

Diallo H, Bamba I, Barima YSS, Visser M, Ballo A, Mama A, Vranken I, Maiga M, Bogaert J. 2011. Effets combinés du climat et des pressions anthropiques sur la dynamique évolutive de la végétation d'une zone protégée du Mali (Réserve de Fina, Boucle du Baoulé).

Sécheresse, 22: 97-107. DOI : 10.1684/sec.2011.0306

Dan Guimbo I, Baragé M, Soumana D. 2012.Etude préliminaire sur l'utilisation alimentaire des plantes spontanées dans les zones périphériques du parc $\mathrm{W}$ du Niger. Int. J. Biol. Chem. Sci., 6(6): 4007-4017.

DOI: https://dx.doi.org /10.4314/ijbcs.v6i6.12

FAO. 2001. State of the world's forests. Rome, 181p.Institut National de la Statistique. 2012. Présentation des résultats globaux définitifs du quatrième (4ème) recensement général de la population et de l'habitat (rgp/h) de 2012.Rapport final, 351p.

Gueguim CD, Tchamba NM, Fotso CR. 2018. Dynamique spatio-temporelle des feux de brousse dans le Parc National du Mbam et Djerem (Cameroun). Int. J. Biol. Chem. Sci., 12(2): 728-748. DOI: https:// dx.doi.org /10.4314/ijbcs.v12i2.10

Kabirou S. 2013. Processus de fixation des populations migrantes à la périphérie de la réserve de Biosphère du $\mathrm{W}$ du Niger. Revue Scientifique Semestrielle Territoires, Sociétés et Environnement, 2: 39-56. URL http://uam.refer.ne/index.php/recherche/ publications- scientifiques/188publication-mars-2015.

Larwanou M. 2005. Dynamiques des ressources environnementales et mutations des systèmes agro-sylvopastoraux en milieu tropical semi-aride: 
le cas de la vallée d'arewa (niger central).Thèse de Doctorat, Université Abdou Moumouni, Niamey, p 266.

Illiassou S A, Diouf A, Mamoudou BM, Sadda AS, Mahamane A, Saadou M. 2015. Dynamics of a third world city: Case of Niamey, Niger. Journal of Geography and Regional Planing, 8 (5): 120-139. DOI: $10.5897 / J G R P 2015.0491$ URL:

http://www.academicjournals.org/JGRP

Mahamadou SM, Boureima A. 2014. Indicateurs de mesure de la pression anthropique sur les Ressources naturelles : exemple de la périphérie du Parc «W Tamou au Niger. VertigO (la revue électronique en science de l'environnement), 14 (1):2-14. DOI: 10.4000/vertigo.14754 URL : http:// vertigo.revues.org/14754 (page consultée le $22 / 09 / 2014$ )

Mama A, Bamba I, Sinsin B, Bogaert J, De Cannière C. 2014. Déforestation, savanisation et développement agricole des paysages de savanes -forets dans la zone soudano- guinéenne du Bénin. Bois et Forêts des Tropiques, 322(4) : $65-75$. DOI : $10.19182 / \mathrm{bft}$ 2014.322.a31237

Mas JF. 2000. Une revue des méthodes et des techniques de télédétection du changement. Canadian Journal of Remote Sensing, 26(4) : 349-362. DOI: 10.1080/07038992. 2000. 10874785 URL :

http://dx.doi.org/10.1080/07038992.2000 .10874785

Moussa MB. 2016. Caractérisation des stades de dégradation des écosystèmes de l'Ouest du Niger et proposition de techniques simples de restauration des stades dégradés. Thèse de Doctorat, Université Abdou Moumouni, Niamey, 166p.

Ministère de l'Eau, de l'Environnement et de la Lutte Contre la Désertification
(MEE/LCD). 2010. Stratégie Nationale et Plan d'Actions pour la conservation durable des éléphants au Niger, Niamey, Niger, 58p.

Schéma Régional d'Aménagement du Territoire de Dosso (SRAT). 2015. Rapport final, Dosso, Niger, p 415.

Sadda AS, Diouf A, Lawali S, Ouedrago M, Bogaert J, Mahamane A. 2016. Pression anthropique et dynamique paysagère en zone rurale semi-aride: Cas de la commune de Tibiri, Région de Maradi (Niger). Tropiculture, 34(2) : 127-139. URL :

https://www.tropicultura.org\%2Fcontent \%2Fv34n2.html\&usg=AOvVaw1ldA8H Fclc RxzXiiYDrmKS (page consulté, le 24/03/2019)

Sitayeb T, Benabdeli K. 2008. Contribution à l'étude de la dynamique de l'occupation des sols de la plaine de la Macta (Algérie) à l'aide de la télédétection et des systèmes d'information géographique. Elsevier Masson SAS. C. R. Biologies, 331 : 466-474. DOI : 10.1016/j.crvi.2008.03.010

Tente ABH. 2005. Recherche sur les facteurs de la diversité floristique des versants du massif de l'Atacora: Secteur Perma-Toucountouna (Bénin), Thèse de Doctorat, Université d'Abomey Calavi, Cotonou, p 252.

Union Internationale pour la Conservation de la Nature (UICN). 2010. Parcs et réserves du Niger: évaluation de l'efficacité de gestion des aires protégées. Ouagadougou, BF : UICN/PACO, 78p. URL http://www.uicn.org/www.papaco.org (page consultée le 28/02/18).

Zean GM, Ahon DB, Koffi BJC. 2018. Peuplement Avifaunique du Campus Universitaire Jean Lorougnon Guedé, Daloa et sa périphérie (Centre Ouest de la Cote d'Ivoire). Int. J. Biol. Chem. Sci., 12(6): 2503-2518. DOI: http://dx.doi 10.4314/ijbcs.v12.i6.4. 